\title{
Removal of Emulsified Oil from Aqueous Environment by Using Polyvinylpyrrolidone-Coated Magnetic Nanoparticles
}

\author{
Shimin Shao ${ }^{1,+}$, Yan $\mathrm{Li}^{1,+}{ }^{+}$, Ting L $\ddot{u}^{1, *}$, Dongming $\mathrm{Qi}^{2}$, Dong Zhang ${ }^{1}\left(\mathbb{D}\right.$ and Hongting Zhao ${ }^{1}$ \\ 1 Institute of Environmental Materials and Applications, College of Materials and Environmental Engineering, \\ Hangzhou Dianzi University, Hangzhou 310018, China; ssmdatiancai@163.com (S.S.); \\ 15700194207@163.com (Y.L.); zhangdong@hdu.edu.cn (D.Z.); info-iem@hdu.edu.cn (H.Z.) \\ 2 Key Laboratory of Advanced Textile Materials and Manufacturing Technology, Ministry of Education, \\ Zhejiang Sci-Tech University, Hangzhou 310018, China; dongmingqi@zstu.edu.cn \\ * Correspondence: lvting@hdu.edu.cn; Tel.: +86-571-87713538 \\ + These authors contributed equally to this work.
}

Received: 10 August 2019; Accepted: 19 September 2019; Published: 24 September 2019

\begin{abstract}
In recent years, a large amount of emulsified oily wastewaters were produced from petroleum and food industries, resulting in severe environmental problems. In this study, a series of polyvinylpyrrolidone (PVP)-coated $\mathrm{Fe}_{3} \mathrm{O}_{4}$ magnetic nanoparticles (MNPs) were prepared via one-step solvothermal method by introducing various amounts or types of PVP. The synthesized MNPs were characterized by multiple techniques, and their demulsification performances were evaluated in petrochemical and vegetable oil wastewaters, respectively. Results showed that the introduction of PVP in solvothermal process could significantly enhance the demulsification efficiency of MNPs, although excessive addition of PVP could not further increase its efficiency. Moreover, the effects of $\mathrm{pH}$, surfactant concentration of wastewater, and the recycle number of MNPs on the demulsification performance were investigated in detail. It was found that the demulsification efficiency decreased with the increase of $\mathrm{pH}$ and surfactant concentration, and the synthetic MNPs were still effective after being reused for 5 cycles under acidic and neutral conditions. It is expected that the development of the PVP-coated MNPs can provide a simple and powerful route for the oily wastewater treatment.
\end{abstract}

Keywords: magnetic nanoparticles; polyvinylpyrrolidone; magnetic separation; demulsification performance; reusability

\section{Introduction}

In recent years, a large amount of emulsified oily wastewaters were produced from petroleum and food industries, resulting in severe environmental problems [1]. The oily wastewater should be strictly treated to meet the requirements of the wastewater discharge. However, the emulsified oil droplets are highly stable due to the presence of surface-active substances, which are either naturally existed or intentionally added [2], making it hard to deal with the emulsified oily wastewaters. Conventional techniques, such as flotation [3], chemical flocculation [4,5] and membrane separation [6-8], were used to break emulsions. However, flotation was ineffective to treat the nanosized oil droplets, chemical flocculation was effective but time-consuming, and membrane separation was limited by the high energy consumption and membrane fouling in actual application [9]. As a consequence, there remains an urgent need to develop easy and powerful techniques or demulsifiers to achieve efficient emulsified oil-water separation.

Recently, oil-water separation by using $\mathrm{Fe}_{3} \mathrm{O}_{4}$ magnetic nanoparticles (MNPs) has attracted considerable attention [10-13], due to its good biocompatibility and easy surface modification. 
The $\mathrm{Fe}_{3} \mathrm{O}_{4}$ MNPs were usually modified with surface-active substances to improve their interfacial activity and water dispersibility. After introduction into the oil-in-water emulsion, the surface-modified $\mathrm{Fe}_{3} \mathrm{O}_{4}$ MNPs can be well dispersed in the aqueous media and then assemble to the emulsified oil-water interface, imparting the magnetic responsiveness to the emulsified droplets $[14,15]$. Thus, the MNPs-coated oil droplets could be rapidly separated from the water phase under an external magnetic field. For example, the MNPs were previously modified with oleic acid [2,11], polyethyleneimine [12], poly(N-isopropylacrylamide) [16,17], poly(2-dimethylaminoethyl methacrylate) [18], (3-aminopropyl)triethoxysilane [14], chitosan [19-21], and so on, and these modified MNPs exhibited good demulsification performance. However, there is still a demand to develop more magnetic demulsifiers and understand the influences of environmental parameters on their demulsification performance.

In the present work, polyvinylpyrrolidone (PVP)-coated $\mathrm{Fe}_{3} \mathrm{O}_{4}$ MNPs were prepared by one-step solvothermal method and used to remove emulsified oil from aqueous environment. Previously, Lead et al. studied the evolution of residual organic component in water phase during the demulsification process by using PVP-coated MNPs; the MNPs showed superior oil removal performance, which was enhanced with increasing ionic strength but reduced in the presence of natural organic macromolecule [22-24]. However, the influences of $\mathrm{pH}$, surfactant and recycle number of MNPs on the demulsification efficiency were not examined; moreover, the effect of PVP in solvothermal reaction system on the demulsification performance of the synthesized MNPs was also not investigated. Consequently, in this study, a series of PVP-coated MNPs were synthesized in the presence of various amounts or types of PVP. The synthesized MNPs were characterized by various techniques, and their demulsification performances were evaluated in petrochemical and vegetable oil wastewaters, respectively. The effects of $\mathrm{pH}$, surfactant concentration on the demulsification performance were investigated in detail. Subsequently, the reusability of the PVP-coated MNPs was further assessed at various $\mathrm{pH}$ levels. It should be noted that larger PVP-coated $\mathrm{Fe}_{3} \mathrm{O}_{4} \mathrm{MNPs}$ were prepared in this work, and different oils and experimental conditions were used as compared with previous study [22-24], and therefore the obtained results cannot be directly compared. However, the influence of the environmental parameters or recycle number of MNPs on the demulsification performances can be still used for reference.

\section{Materials and Methods}

\subsection{Materials}

Iron chloride hexahydrate $\left(\mathrm{FeCl}_{3} \cdot 6 \mathrm{H}_{2} \mathrm{O}\right)$, anhydrous sodium acetate $(\mathrm{NaAc})$, ethylene glycol (EG), n-hexane, sodium dodecyl sulfate (SDS), and sodium hydroxide $(\mathrm{NaOH})$ were purchased from Aladdin Chemistry. Polyvinylpyrrolidone (PVP, with a molecular weight of $10 \mathrm{k}$ and $1300 \mathrm{k}$, respectively) was purchased from Shanghai Macklin Biochemical Co. Ltd (Shanghai, China). Hydrochloric acid (HCl) was supplied by Zhejiang Sanying chemical reagent Co. Ltd (Lanxi, China). Ethanol was purchased from Hangzhou Gaojing fine chemical Co. Ltd (Hangzhou, China). A commercially available diesel was obtained from Sinopec, and the soybean oil was obtained from supermarket. Deionized water was used throughout the experiment. All chemicals were of analytical grade and used without further purification.

\subsection{Synthesis of $\mathrm{Fe}_{3} \mathrm{O}_{4} \mathrm{MNPS}$}

$\mathrm{Fe}_{3} \mathrm{O}_{4} \mathrm{MNPs}$ were prepared by a modified solvothermal method [25]. Briefly, a certain amount of $\mathrm{FeCl}_{3} \cdot 6 \mathrm{H}_{2} \mathrm{O}$, NaAc and PVP were completely dissolved in EG (Table 1). The resultant solution was then introduced into a Teflon-lined autoclave and kept at $200^{\circ} \mathrm{C}$ for $6 \mathrm{~h}$. The product was collected and washed with ethanol and water three times. Subsequently, a portion of synthetic $\mathrm{Fe}_{3} \mathrm{O}_{4} \mathrm{MNPs}$ was dried at $50{ }^{\circ} \mathrm{C}$ under vacuum for $24 \mathrm{~h}$, and the rest of MNPs were dispersed in water for further use. 
Table 1. Recipes for the synthesis of PVP-coated $\mathrm{Fe}_{3} \mathrm{O}_{4}$ MNPs.

\begin{tabular}{ccccc}
\hline Sample Number & $\mathrm{FeCl}_{\mathbf{3}} \cdot \mathbf{6} \mathbf{H}_{\mathbf{2}} \mathbf{O}(\mathbf{g})$ & $\mathbf{N a A c}(\mathrm{g})$ & $\mathbf{P V P}(\mathrm{g})$ & $\mathbf{E G}(\mathbf{m L})$ \\
\hline S0 & 2.0 & 6.0 & 0 & 65 \\
$\mathrm{~S} 1$ & 2.0 & 6.0 & $1.5(10 \mathrm{k})$ & 65 \\
$\mathrm{~S} 2$ & 2.0 & 6.0 & $3.0(10 \mathrm{k})$ & 65 \\
$\mathrm{~S} 3$ & 2.0 & 6.0 & $4.5(10 \mathrm{k})$ & 65 \\
$\mathrm{~S} 4$ & 2.0 & 6.0 & $1.5(1300 \mathrm{k})$ & 65 \\
\hline
\end{tabular}

\subsection{Characterization}

Size and morphology of MNPs were observed by transmission electron microscopy (TEM, JSM-1200EX, Tokyo, Japan) with a voltage of $80 \mathrm{kv}$. The MNPs was dispersed in neutral deionized water, and the concentration was kept at about $0.2 \mathrm{~g} / \mathrm{L}$; thereafter, a drop of nanoparticle dispersion was cast on the copper net and died at room temperature for TEM examination. Zeta potential of MNPs and oil droplets at various $\mathrm{pH}$ levels was measured with a zeta potential analyzer (Nano ZS90, Malvern Instruments Company, Malvern, UK), the nanoparticle or oil droplet concentration of aqueous dispersion was also kept at around $0.2 \mathrm{~g} / \mathrm{L}$. Herein, dried samples of MNPs were used throughout the characterization of X-ray diffraction (XRD), Fourier transform infrared spectroscopy (FTIR), Thermogravimetric analysis (TGA), and physical property measurement system (PPMS). X-ray powder diffraction patterns were determined by using an X-ray diffractometer (XRD, D8 Discover) in the $2 \theta$ range of $10^{\circ}-90^{\circ}$. FTIR of MNPs was performed using a FTIR spectrometer (Nicolet 6700 , Thermo Fisher Scientific, Boston, MA, USA) from 400 to $4000 \mathrm{~cm}^{-1}$, and the samples were prepared by using $\mathrm{KBr}$ pellet technique. TGA was performed on a thermal gravimetric analyzer (TGA/DSC 1, Mettler Toledo, Zurich, Swit) under nitrogen atmosphere from 25 to $800{ }^{\circ} \mathrm{C}$ at a heating rate of $10^{\circ} \mathrm{C} / \mathrm{min}$. Magnetization measurements were taken on a PPMS (PPMS-9, Quantum Design, San Diego, CA, USA) at room temperature; prior to examination, the nanoparticle power was bonded with each other to form a globule by using adhesive, and its size was no more than $3 \mathrm{~mm}$. Water transmittance of emulsion before and after oil separation was determined by means of a UV-vis spectrometer (UV-2450, Shimadzu, Kyoto, Kyoto Prefecture, Japan) at a wavelength of $610 \mathrm{~nm}$. The microscopic image of the mixture of emulsion and MNPs was examined by using a digital optical microscope (KH-7700, Hirox, Tokyo, Japan).

\subsection{Demulsification Test}

Oil-in-water emulsion was prepared by powerful sonication under magnetic stirring for $5 \mathrm{~min}$, and the concentration of diesel or soybean oil was kept at $2.7 \mathrm{~g} / \mathrm{L}$. A certain amount of SDS was introduced following by short ultrasound treatment to make majority of surfactant assemble at the oil-water interface. $\mathrm{pH}$ of emulsion was regulated by using $\mathrm{HCl}$ or $\mathrm{NaOH}$ solution. The SDS concentration and $\mathrm{pH}$ were kept at $0 \mathrm{~mol} / \mathrm{L}$ and 7.0, respectively, except as otherwise indicated. The demulsification experiment was performed at room temperature. A certain amount of MNPs were added to the emulsion, and the mixture was shaken just by hand (without sonication) to let the MNPs attach to the oil droplets. The MNPs-coated oil droplets were rapidly separated by using a magnet within $5 \mathrm{~min}$. The water transmittance after magnetic separation was determined to assess the demulsification efficiency. The initial transmittance of emulsion was closed to zero.

\subsection{Recycle Tests}

After the demulsification test, the spent MNPs were regenerated by washing with ethanol to remove the attached diesel oil and washing with n-hexane and ethanol to remove the attached soybean oil. The regenerated MNPs were re-dispersed in water and then reused in the next cycle of demulsification test. The recycle of MNPs was carried out for 7 times to evaluate their recyclability. 


\section{Results and Discussion}

\subsection{Characterizations}

TEM images of PVP-coated $\mathrm{Fe}_{3} \mathrm{O}_{4}$ MNPs are shown in Figure 1. Without addition of PVP, the synthesized $\mathrm{Fe}_{3} \mathrm{O}_{4}$ MNPs could not be well dispersed in water. However, after the introduction of PVP (1.5 g) into the solvothermal system, the water dispersibility of synthetic MNPs was significantly improved. As shown in Figure 1a, the spherical MNPs were polydisperse, and their size ranged from about 90 to $410 \mathrm{~nm}$. XRD patterns of the synthesized MNPs are shown in Figure 2a. Diffraction peaks at $2 \theta$ values of $30.3^{\circ}, 35.5^{\circ}, 43.2^{\circ}, 53.6^{\circ}, 57.3^{\circ}$, and $62.9^{\circ}$ can be indexed to the indices (220), (311), (400), (422), (511), and (440) of standard $\mathrm{Fe}_{3} \mathrm{O}_{4}$ [25], suggesting the successful preparation of $\mathrm{Fe}_{3} \mathrm{O}_{4}$ MNPs. Meanwhile, no obvious difference of diffraction peaks was observed between bare $\mathrm{Fe}_{3} \mathrm{O}_{4}$ and PVP-coated $\mathrm{Fe}_{3} \mathrm{O}_{4}$ MNPs, indicating that the crystal structure of $\mathrm{Fe}_{3} \mathrm{O}_{4}$ was not varied after PVP addition.
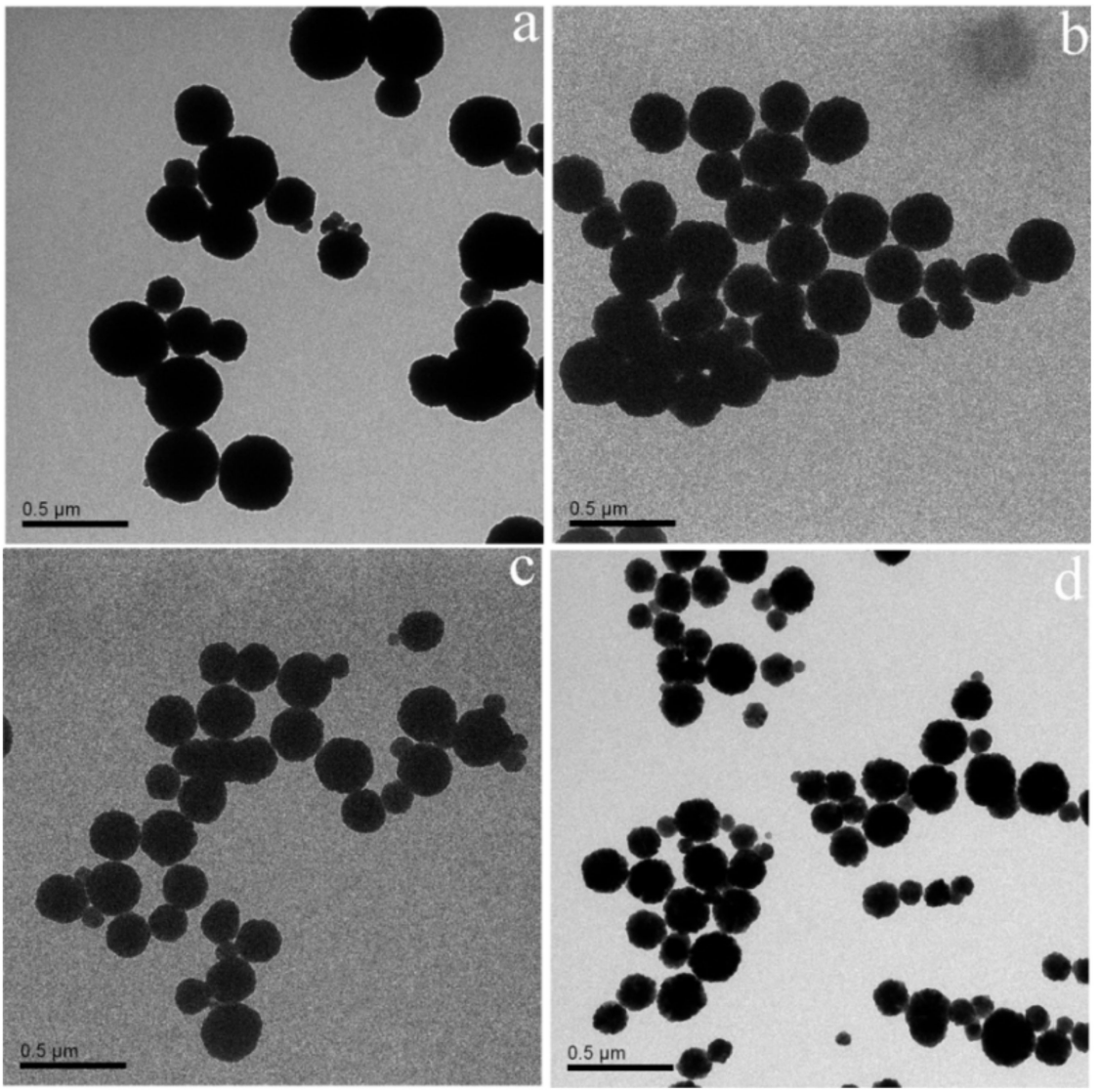

Figure 1. TEM images of PVP-coated $\mathrm{Fe}_{3} \mathrm{O}_{4}$ MNPs ((a): S1; (b): S2; (c): S3; (d): S4).

Figure $2 \mathrm{~b}$ shows the FTIR spectra of PVP, bare $\mathrm{Fe}_{3} \mathrm{O}_{4}$ and PVP-coated $\mathrm{Fe}_{3} \mathrm{O}_{4} \mathrm{MNPs}$. The intense absorption peak at around $599 \mathrm{~cm}^{-1}$ corresponded to the $\mathrm{Fe}-\mathrm{O}$ vibrations [20]. As compared with the bare $\mathrm{Fe}_{3} \mathrm{O}_{4}$, the stretching vibration of $\mathrm{C}=\mathrm{O}$ in PVP-coated $\mathrm{Fe}_{3} \mathrm{O}_{4} \mathrm{MNPs}$ moved from 1629 to $1657 \mathrm{~cm}^{-1}$; moreover, the peak at around $1290 \mathrm{~cm}^{-1}$ ascribed to the vibrations of C-N appeared, and the peak at around $2936 \mathrm{~cm}^{-1}$ due to the $\mathrm{C}$-H stretching became notable in PVP-coated $\mathrm{Fe}_{3} \mathrm{O}_{4} \mathrm{MNPs}_{\text {. These results }}$ confirmed that PVP was indeed coated on the surface of $\mathrm{Fe}_{3} \mathrm{O}_{4}$. In order to estimate the PVP content in the synthetic MNPs, TGA experiment was performed on the bare $\mathrm{Fe}_{3} \mathrm{O}_{4}$ and PVP-coated $\mathrm{Fe}_{3} \mathrm{O}_{4} \mathrm{MNPs}$ (Figure 2c). The evaporation of physically absorbed water was usually found below $120^{\circ} \mathrm{C}$, while the decomposition of organic component was mainly observed between 120 and $650{ }^{\circ} \mathrm{C}$ [22]. 

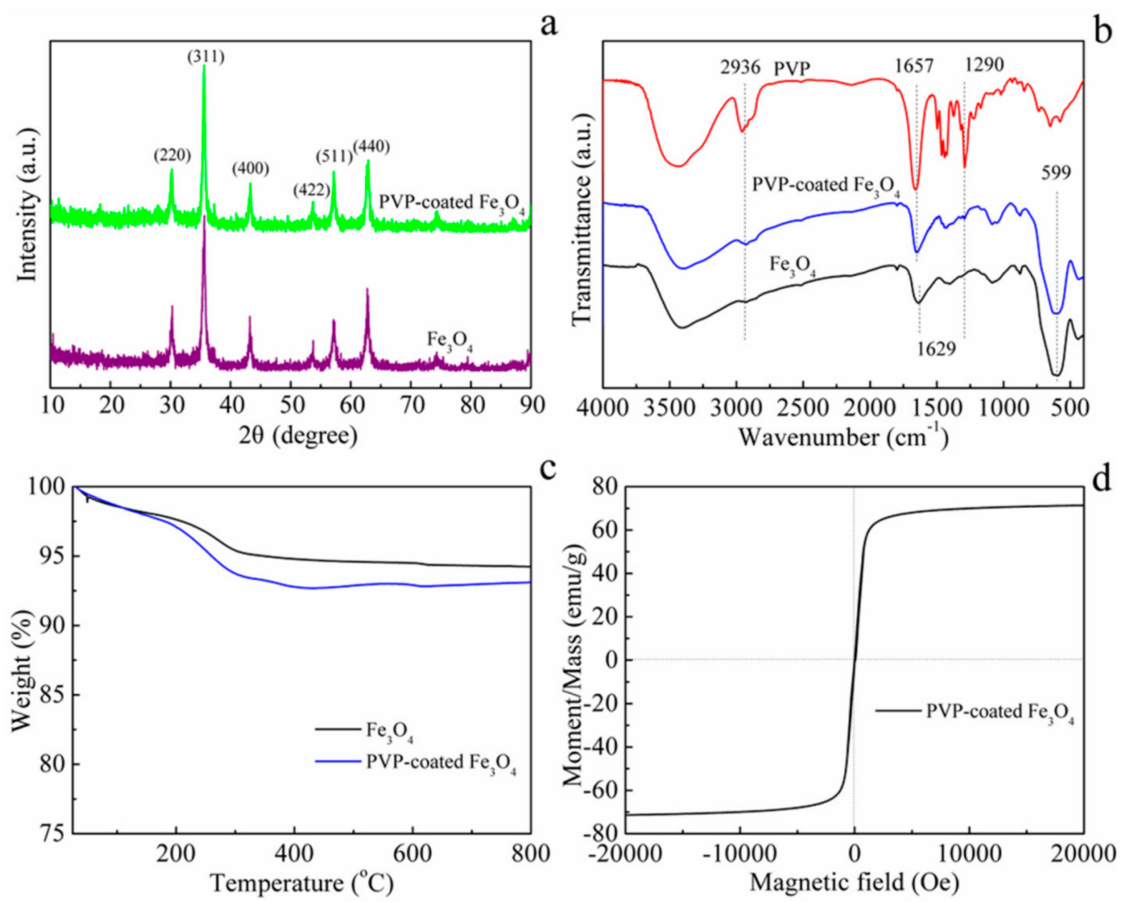

Figure 2. XRD patterns (a), FTIR spectra (b), TGA curves (c), and magnetic hysteresis loop (d) of the synthesized MNPs $\left(\mathrm{Fe}_{3} \mathrm{O}_{4}\right.$ : S0, PVP-coated $\mathrm{Fe}_{3} \mathrm{O}_{4}$ : S1).

Obviously, the weight loss of MNPs increased after introduction of PVP, and the increase in organic component decomposition could be attributed to the PVP coated on $\mathrm{Fe}_{3} \mathrm{O}_{4} \mathrm{MNPs}$. Accordingly, the PVP content was estimated to be about $1.4 \%$ based on the weight of $\mathrm{Fe}_{3} \mathrm{O}_{4}$ MNPs. Furthermore, the magnetic property of MNPs was measured at room temperature (Figure 2d).

The saturation magnetization of PVP-coated $\mathrm{Fe}_{3} \mathrm{O}_{4}$ MNPs was determined to be $71.4 \mathrm{e} \mathrm{mu} / \mathrm{g}$; meanwhile, negligible hysteresis of magnetism was observed. The superparamagnetic behavior can be explained by the fact that each $\mathrm{Fe}_{3} \mathrm{O}_{4}$ microsphere prepared via solvothermal method was composed of many nanosized primary particles, which are usually smaller than $30 \mathrm{~nm}$ [18]. This result indicated that the synthesized PVP-coated $\mathrm{Fe}_{3} \mathrm{O}_{4}$ MNPs could be well dispersed in water in the absence of magnetic field and rapidly collected from aqueous media by applying magnetic field.

\subsection{Demulsification Performance}

Figure 3 shows the demulsification performance of the $\mathrm{Fe}_{3} \mathrm{O}_{4}$ MNPs prepared with various amounts or types of PVP. As mentioned earlier, the bare $\mathrm{Fe}_{3} \mathrm{O}_{4}$ MNPs could not be equably dispersed in water and was too hydrophilic to attach to the oil droplets [2,12], thereby showing negligible demulsification effect. After introduction of $1.5 \mathrm{~g}$ PVP, the synthesized PVP-coated $\mathrm{Fe}_{3} \mathrm{O}_{4} \mathrm{MNPs}$ exhibited good dispersibility and certain interfacial activity due to the amphiphilic nature of PVP [26], and hence their demulsification efficiencies were significantly enhanced. When the PVP amount increased from 1.5 to $4.5 \mathrm{~g}$ or the PVP molecular weight increased from $10 \mathrm{k}$ to $1300 \mathrm{k}$, the size of synthesized PVP-coated $\mathrm{Fe}_{3} \mathrm{O}_{4}$ MNPs decreased slightly (Figure 1); however, their demulsification effects were not further enhanced and nearly kept constant (Figure 3). As a result, the PVP-coated $\mathrm{Fe}_{3} \mathrm{O}_{4}$ MNPs prepared with addition of $1.5 \mathrm{~g}$ PVP (10k) were further applied to treat the petrochemical and vegetable oil wastewaters under various conditions. 


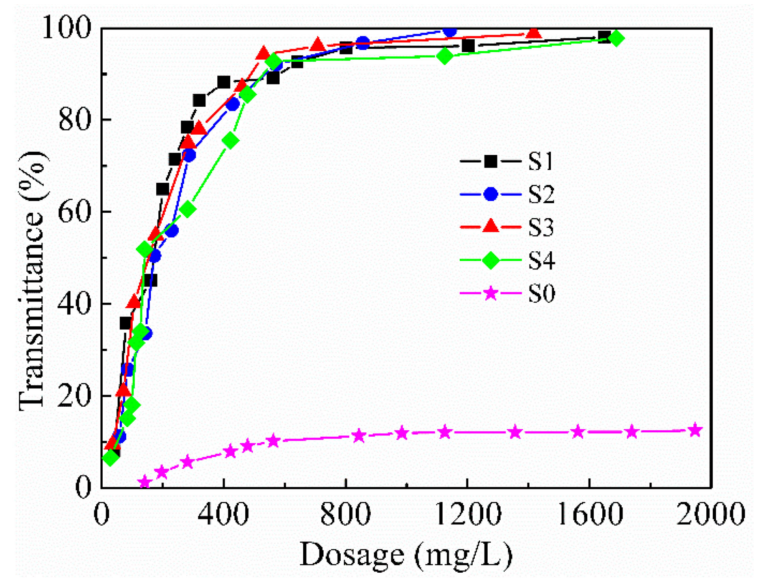

Figure 3. Demulsification effect of various $\mathrm{Fe}_{3} \mathrm{O}_{4}$ MNPs in diesel oil-in-water emulsion.

$\mathrm{pH}$ is usually regarded as an important parameter affecting the oily wastewater treatment efficiency [4], and hence the demulsification performance of the MNPs was evaluated at various $\mathrm{pH}$ levels. Figure 4 shows the micrographs of the diesel (soybean) oil-in-water emulsion and its mixture with MNPs at $\mathrm{pH}$ 4.0, 7.0, and 10.0, respectively. The emulsified oil droplets with a size of less than $10 \mu \mathrm{m}$ could be easily observed in the microscopic image (Figure 4a,e). However, after addition of MNPs to the emulsion, free oil droplets almost disappeared in the microscopic view; instead, some aggregated MNPs were found under different $\mathrm{pH}$ conditions (Figure $4 \mathrm{~b}-\mathrm{d}, \mathrm{f}-\mathrm{h}$ ). The disappearance of free oil droplets and aggregated morphology of MNPs indicated the oil sorption onto the MNPs [24]. In other words, the synthesized PVP-coated $\mathrm{Fe}_{3} \mathrm{O}_{4}$ MNPs attaches to the oil droplets once it is added to the emulsion. Therefore, the emulsified oil droplets could be rapidly separated from the water phase by applying magnetic field (Figure $4 \mathrm{i}-\mathrm{n}$ ). The detailed effect of $\mathrm{pH}$ on the demulsification efficiency was shown in Figure 5a,b. It was found that the demulsification efficiency declined with $\mathrm{pH}$ rising in both diesel and soybean oil-in-water emulsions. As shown in Figure 6, both emulsified oil droplets and PVP-coated $\mathrm{Fe}_{3} \mathrm{O}_{4}$ MNPs were negatively charged at various $\mathrm{pH}$ levels, and hence, the electrostatic interaction between MNPs and oil droplets was repulsive. However, the PVP-coated MNPs could still attach to the oil droplets mainly via hydrophobic effect, which was also proposed by Lead et al. [23]. The zeta potential decreased with $\mathrm{pH}$ rising (Figure 6), and therefore the electrostatic repulsion between MNPs and oil droplets became stronger at high $\mathrm{pH}$ level. Thus, the MNPs became difficult to attach to the oil droplets, resulting in a reduction in demulsification efficiency. Besides, it can be seen in Figure $5 a, b$ that the demulsification efficiency did not show significant difference between diesel oil-in-water emulsion and soybean oil-in-water emulsion at the same dosage of PVP-coated MNPs. When the dosage reached $400 \mathrm{mg} / \mathrm{L}$ at $\mathrm{pH} 7.0$, the water transmittance exceeded $85 \%$, indicating the oil removal ratio exceeded 99\% [12]. In other words, the PVP-coated MNPs are capable of effectively removing emulsified oil from a synthetic wastewater (2.7 g/L oil) at a ratio (oil:MNPs) of 6.75:1 under neutral conditions. The oil absorption capacity of synthetic PVP-coated MNPs seems to be lower than that in previous reports [22-24], presumably due to the much larger size of MNPs and shorter magnetic separation time in this study. 


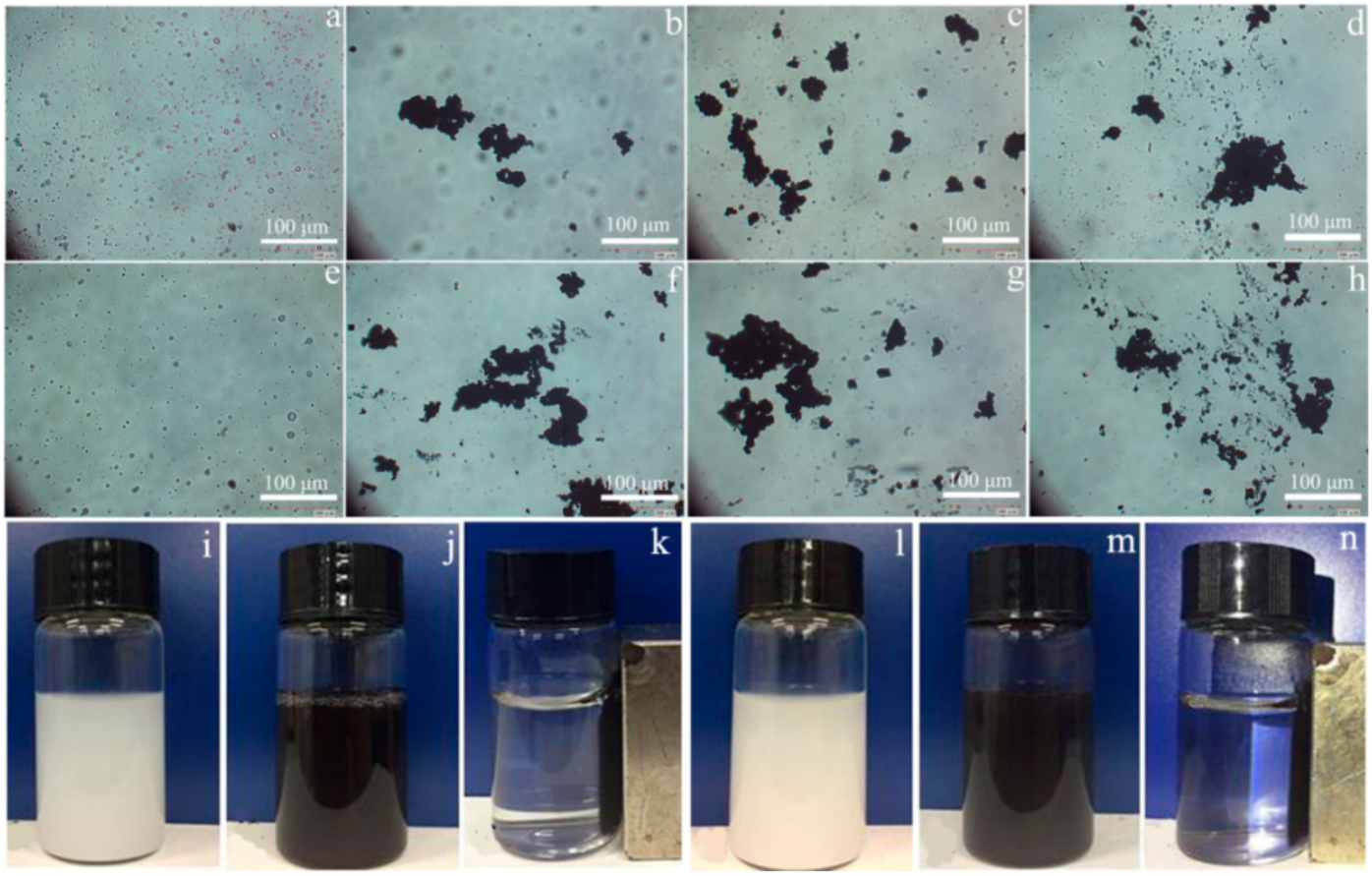

Figure 4. Microscopic images of diesel oil-in-water emulsion (a) and its mixture with MNPs (S1) at $\mathrm{pH}$ 4.0 (b), 7.0 (c), and 10.0 (d); soybean oil-in-water emulsion (e) and its mixture with MNPs (S1) at pH 4.0 (f), 7.0 (g), and 10.0 (h); photographs of diesel oil-in-water emulsion (i), its mixture with MNPs (S1) at $\mathrm{pH} 7.0(\mathbf{j})$ and water solution after magnetic separation $(\mathbf{k})$; photographs of soybean oil-in-water emulsion (1), its mixture with MNPs (S1) at pH 7.0 (m) and water solution after magnetic separation (n).
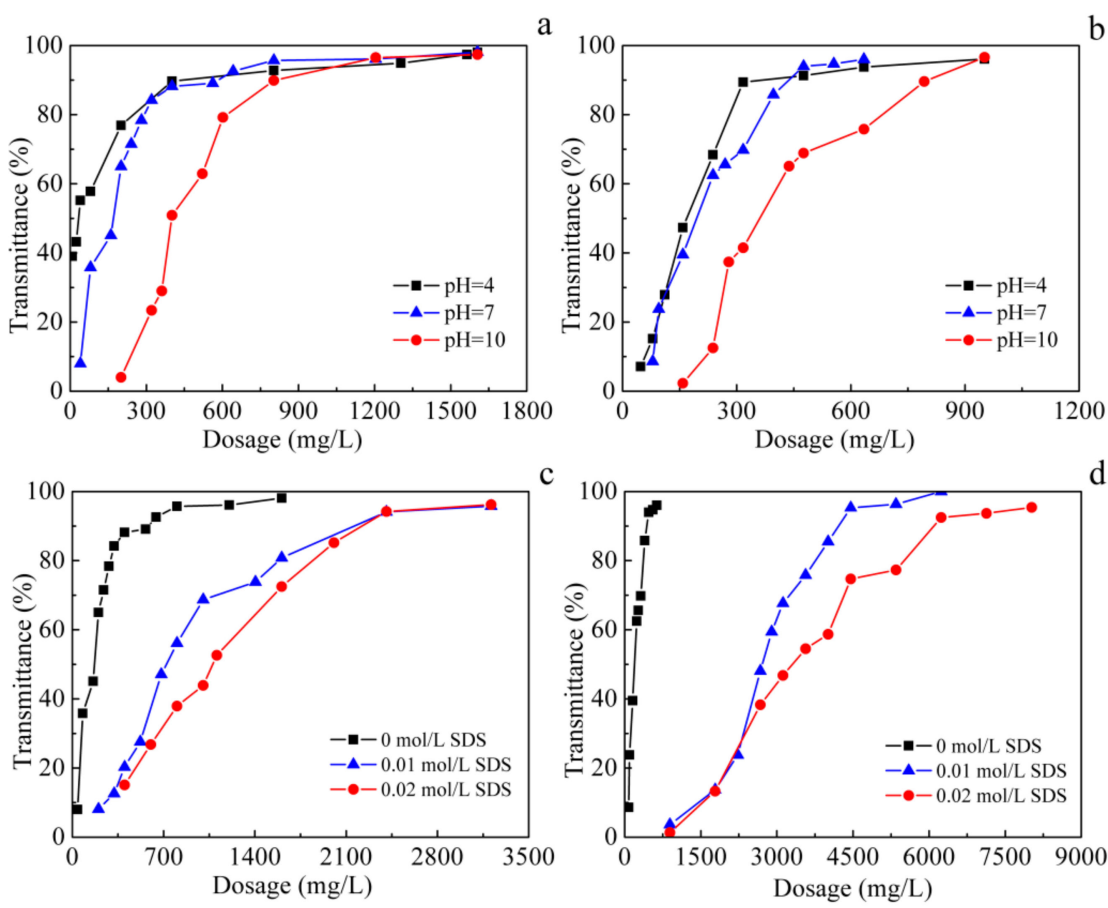

Figure 5. Demulsification effect of MNPs (S1) at various $\mathrm{pH}$ levels in diesel oil-in-water emulsion (a) and soybean oil-in-water emulsion (b); demulsification effect of MNPs (S1) at various SDS concentrations in diesel oil-in-water emulsion (c) and soybean oil-in-water emulsion (d). 


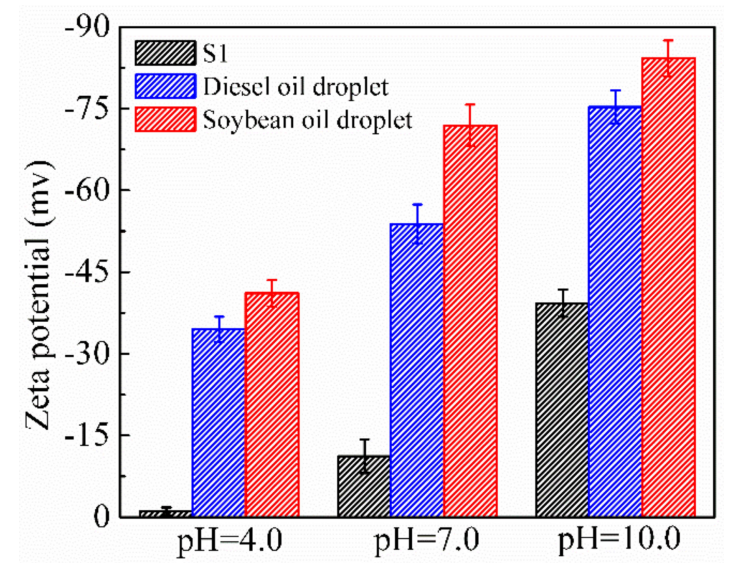

Figure 6. Zeta potential of MNPs (S1) and oil droplets at various $\mathrm{pH}$ levels.

Emulsified oily wastewater usually contains surface-active substances, which either naturally exist or are intentionally added [2]. Therefore, it is important to examine the influence of surfactant on the demulsification performance. Herein, SDS was used as the surface-active substance. As shown in Figure $5 c, d$, the demulsification efficiency decreased significantly with the addition of SDS in both diesel and soybean oil-in-water emulsions. It is well known that the oil droplet was attached to PVP-coated MNPs via hydrophobic interaction [23]. After introduction of SDS, majority of SDS would be distributed on the surface of oil droplets; meanwhile, part of SDS might also attach to PVP-coated MNPs, since the SDS molecule would be bound to a hydrophobic site of PVP molecules due to the attractive force of the hydrophobic association [27]. The SDS adsorption not only increased the hydrophilicity of the oil, thereby decreasing the hydrophobic driver but also increased the electrostatic repulsion between MNPs and oil droplets. Both factors did not favor the sorption of oil to PVP-coated MNPs, and therefore, their demulsification effect declined remarkably with addition of surfactant. This phenomenon is similar to the impact of natural organic macromolecules on the oil separation efficiency as previously studied [23].

\subsection{Recyclability of MNPs}

As compared with traditional flocculants and demulsifiers, another advantage is that the MNPs can be recycled in addition to their high demulsification efficiency. The reusability of PVP-coated $\mathrm{Fe}_{3} \mathrm{O}_{4}$ MNPs was evaluated by treating the diesel (soybean) oil-in-water emulsion at various $\mathrm{pH}$ levels (Figure 7). It was found that the water transmittance still exceeded $60 \%$ after 5 cycles of magnetic demulsification under acidic and neutral conditions. According to our previous study [12], the oil removal ratio was higher than $95 \%$ when the water transmittance reached $60 \%$, suggesting that the PVP-coated $\mathrm{Fe}_{3} \mathrm{O}_{4}$ MNPs were still efficient after 5 cycles at low and middle pH levels. However, under alkaline condition, the demulsification efficiency declined dramatically during the reutilization process. This can be presumably explained as follows. As discussed earlier, electrostatic repulsion was the negative factor for oil absorption onto MNPs. During the recycle process of MNPs, some substances in wastewater would partly contaminate the MNPs, thereby competitively blocking the potential for oil sorption to some extent. At this time, the effect of negative factor (electrostatic repulsion) on the oil attachment to MNPs was significantly magnified. As indicated by the zeta potentials, the electrostatic repulsion between oil droplets and MNPs at high $\mathrm{pH}$ level was much stronger than that at low and middle $\mathrm{pH}$ levels. Therefore, the PVP-coated $\mathrm{Fe}_{3} \mathrm{O}_{4} \mathrm{MNPs}$ exhibited evident slowdown in demulsification performance under alkaline condition during their recycle process. Besides, as mentioned earlier, the PVP-coated MNPs are able to remove emulsified oil at a ratio (oil:MNPs) of 6.75:1 at medium $\mathrm{pH}$ level. Accordingly, $80 \mathrm{~g}$ of MNPs was required to treat a ton of synthetic emulsified oily wastewater $(2.7 \mathrm{~g} / \mathrm{L}$ oil, without surfactant) in this laboratory, assuming that the MNPs can be recycled for 5 times. 

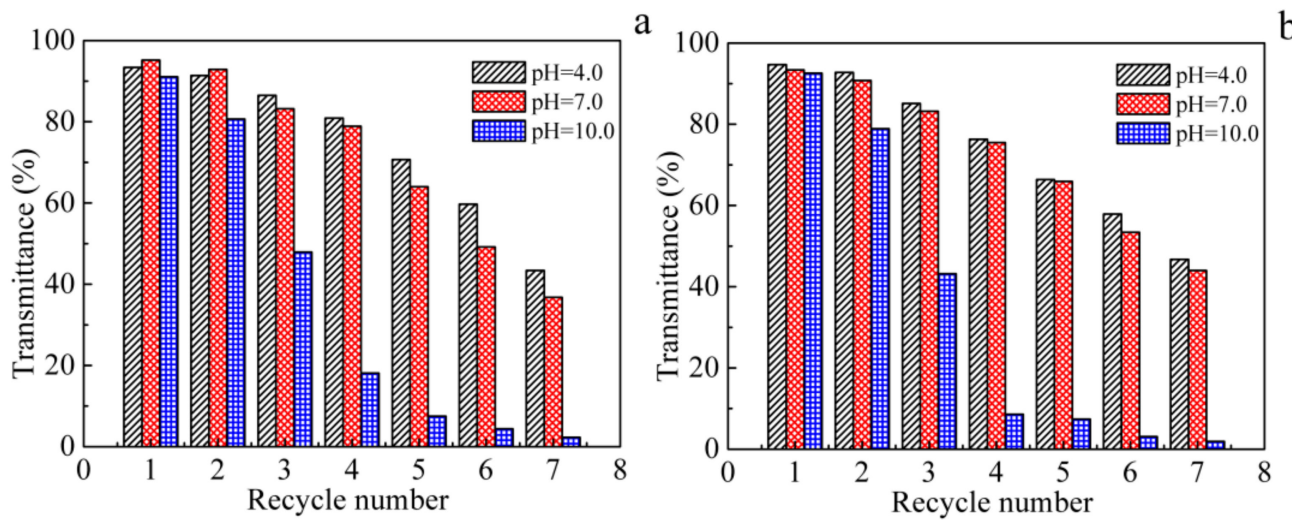

Figure 7. Reusability of MNPs (S1) at various pH levels in diesel oil-in-water emulsion (a) and soybean oil-in-water emulsion (b).

\section{Conclusions}

In summary, the PVP-coated $\mathrm{Fe}_{3} \mathrm{O}_{4}$ MNPs prepared via solvothermal method exhibited good demulsification performance toward treating petrochemical and vegetable oil wastewaters. Demulsification efficiency of MNPs was significantly enhanced due to the surface coating with PVP. Increasing PVP content or molecular weight in solvothermal process resulted in a slight decrease in nanoparticle size, whereas their demulsification performance was not further improved and nearly kept constant. Moreover, $\mathrm{pH}$ and surfactant significantly influence the demulsification performance of PVP-coated $\mathrm{Fe}_{3} \mathrm{O}_{4}$ MNPs. It was found that their demulsification efficiency decreased markedly with increasing $\mathrm{pH}$ or surfactant concentration. Recycle experiment indicated that PVP-coated $\mathrm{Fe}_{3} \mathrm{O}_{4} \mathrm{MNPs}$ showed good recyclability under acidic and neutral conditions but poor reusability under alkaline conditions. It is believed that use of PVP-coated MNPs is a facile and reliable technique for removing emulsified oil from aqueous environment at low and medium $\mathrm{pH}$ conditions.

Author Contributions: Nanoparticles synthesis and water treatment, S.S. and Y.L.; nanoparticle characterization, T.L. and D.Q.; manuscript preparation, T.L.; manuscript review and editing, D.Z. and H.Z.; S.S. and Y.L. contributed equally to this work.

Funding: This research was funded by the National Natural Science Foundation of China (NNSFC) project (\#21878064), and the Natural Science Foundation of Zhejiang Province (\#LZ18E030002).

Acknowledgments: We greatly appreciate anonymous reviewer's constructive comments which helped to improve the quality of our manuscript.

Conflicts of Interest: The authors declare no conflict of interest.

\section{References}

1. Bratskaya, S.; Avramenko, V.; Schwarz, S.; Philippov, A. Enhanced flocculation of oil-in-water emulsions by hydrophobically modified chitosan derivatives. Colloids Surf. A Physicochem. Eng. Asp. 2006, 275, 168-176. [CrossRef]

2. Liang, J.; Du, N.; Song, S.; Hou, W. Magnetic demulsification of diluted crude oil-in-water nanoemulsions using oleic acid-coated magnetite nanoparticles. Colloids Surf. A Physicochem. Eng. Asp. 2015, 466, 197-202. [CrossRef]

3. Rajak, V.K.; Relish, K.K.; Kumar, S.; Mandal, A. Mechanism and kinetics of separation of oil from oil-in-water emulsion by air flotation. Petrol. Sci. Technol. 2016, 33, 1861-1868. [CrossRef]

4. Lü, T.; Luo, C.; Qi, D.; Zhang, D.; Zhao, H. Efficient treatment of emulsified oily wastewater by using amphipathic chitosan-based flocculant. React. Funct. Polym. 2019, 139, 133-141. [CrossRef]

5. Zhang, H.; Duan, M.; Fang, S.; Zhai, L.; Wang, X. Preparation of a novel flocculant and its performance for treating acidic oily wastewater. RSC Adv. 2016, 6, 106102-106108. [CrossRef]

6. Zhao, Y.; Zhang, M.; Wang, Z. Underwater superoleophobic membrane with enhanced oil-water separation, antimicrobial, and antifouling activities. Adv. Mater. Interfaces 2016, 3, 1500664. [CrossRef] 
7. Wang, Z.; Liu, G.; Huang, S. In situ generated janus fabrics for the rapid and efficient separation of oil from oil-in-water emulsions. Angew. Chem. Int. Ed. 2016, 55, 14610-14613. [CrossRef]

8. Lv, W.; Mei, Q.; Xiao, J.; Du, M.; Zheng, Q. 3D multiscale superhydrophilic sponges with delicately designed pore size for ultrafast oil/water separation. Adv. Funct. Mater. 2017, 27, 1704293. [CrossRef]

9. Xu, H.; Jia, W.; Ren, S.; Wang, J. Novel and recyclable demulsifier of expanded perlite grafted by magnetic nanoparticles for oil separation from emulsified oil wastewaters. Chem. Eng. J. 2018, 337, 10-18. [CrossRef]

10. Mirshahghassemi, S.; Ebner, A.D.; Cai, B.; Lead, J.R. Application of high gradient magnetic separation for oil remediation using polymer-coated magnetic nanoparticles. Sep. Purif. Technol. 2017, 179, 328-334. [CrossRef]

11. Liang, J.; Li, H.; Yan, J.; Hou, W. Demulsification of oleic-acid-coated magnetite nanoparticles for cyclohexane-in-water nanoemulsions. Energy Fuels 2014, 28, 6172-6178. [CrossRef]

12. Lü, T.; Qi, D.; Zhang, D.; Lü, Y.; Zhao, H. A facile method for emulsified oil-water separation by using polyethylenimine-coated magnetic nanoparticles. J. Nanopart. Res. 2018, 20, 88. [CrossRef]

13. Liang, C.; He, X.; Liu, Q.; Xu, Z. Adsorption-based synthesis of magnetically responsive and interfacially active composite nanoparticles for dewatering of water-in-diluted bitumen emulsions. Energy Fuels 2018, 32, 8078-8089. [CrossRef]

14. Lü, T.; Zhang, S.; Qi, D.; Zhang, D.; Vancec, G.F.; Zhao, H. Synthesis of pH-sensitive and recyclable magnetic nanoparticles for efficient separation of emulsified oil from aqueous environments. Appl. Surf. Sci. 2017, 396, 1604-1612. [CrossRef]

15. Zhang, J.; Li, Y.; Bao, M.; Yang, X.; Wang, Z. Facile fabrication of cyclodextrin-modified magnetic particles for effective demulsification from various types of emulsions. Environ. Sci. Technol. 2016, 50, 8809-8816. [CrossRef]

16. Lü, T.; Zhang, S.; Qi, D.; Zhang, D.; Zhao, H. Thermosensitive poly(N-isopropylacrylamide)-grafted magnetic nanoparticles for efficient treatment of emulsified oily wastewater. J. Alloys Compd. 2016, 688, 513-520. [CrossRef]

17. Chen, Y.; Bai, Y.; Chen, S.; Ju, J.; Li, Y.; Wang, T.; Wang, Q. Stimuli-responsive composite particles as solid-stabilizers for effective oil harvesting. ACS Appl. Mater. Interfaces 2014, 6, 13334-13338. [CrossRef]

18. Wang, X.; Shi, Y.; Graff, R.W.; Lee, D.; Gao, H. Developing recyclable pH-responsive magnetic nanoparticles for oil-water separation. Polymer 2015, 72, 361-367. [CrossRef]

19. Lü, T.; Zhang, S.; Qi, D.; Zhang, D.; Zhao, H. Enhanced demulsification from aqueous media by using magnetic chitosan-based flocculant. J. Colloid Interfaces Sci. 2018, 518, 76-83. [CrossRef]

20. Lü, T.; Chen, Y.; Qi, D.; Cao, Z.; Zhang, D.; Zhao, H. Treatment of emulsified oil wastewaters by using chitosan grafted magnetic nanoparticles. J. Alloys Compd. 2017, 696, 1205-1212. [CrossRef]

21. Zhang, S.; Lü, T.; Qi, D.; Cao, Z.; Zhang, D.; Zhao, H. Synthesis of quaternized chitosan-coated magnetic nanoparticles for oil-water separation. Mater. Lett. 2017, 191, 128-131. [CrossRef]

22. Mirshahghassemi, S.; Lead, J.R. Oil recovery from water under environmentally relevant conditions using magnetic nanoparticles. Environ. Sci. Technol. 2015, 49, 11729-11736. [CrossRef]

23. Mirshahghassemi, S.; Cai, B.; Lead, J.R. Evaluation of polymer-coated magnetic nanoparticles for oil separation under environmentally relevant conditions: Effect of ionic strength and natural organic macromolecules. Environ. Sci. Nano 2016, 3, 780-787. [CrossRef]

24. Mirshahghassemi, S.; Lead, J.R. A facile and cost-effective method for separation of oil-water mixtures using polymer-coated iron oxide nanoparticles. Environ. Sci. Technol. 2014, 48, 14558-14563.

25. Song, H.; Liu, M.; Li, S.; Chen, L.; Lin, C.; Zhang, L. Polyvinyl pyrrolidone-assisted solvothermal synthesis of $\mathrm{Fe}_{3} \mathrm{O}_{4}$ vesicular nanospheres. J. Nanosci. Nanotechnol. 2015, 15, 3998-4002. [CrossRef]

26. Li, L.; Nicholas, R.J.; Chen, C.; Darton, R.C.; Baker, S.C. Comparative study of photoluminescence of single-walled carbon nanotubes wrapped with sodium dodecyl sulfate, surfactin and polyvinylpyrrolidone. Nanotechnology 2005, 16, S202-S205. [CrossRef]

27. Shen, Q.; Wei, H.; Wang, L.; Zhou, Y.; Zhao, Y.; Zhang, Z.; Wang, D.; Xu, G.; Xu, D. Crystallization and aggregation behaviors of calcium carbonate in the presence of poly(vinylpyrrolidone) and sodium dodecyl sulfate. J. Phys. Chem. B 2005, 109, 18342-18347. [CrossRef]

(C) 2019 by the authors. Licensee MDPI, Basel, Switzerland. This article is an open access article distributed under the terms and conditions of the Creative Commons Attribution (CC BY) license (http://creativecommons.org/licenses/by/4.0/). 